\title{
ANÁLISIS TEMÁTICO DE LA INVESTIGACIÓN, DESARROLLO E INNOVACIÓN DENTRO DE LA GESTIÓN EMPRESARIAL Y ADMINISTRACIÓN DE EMPRESAS DESDE 2015 A 2019
}

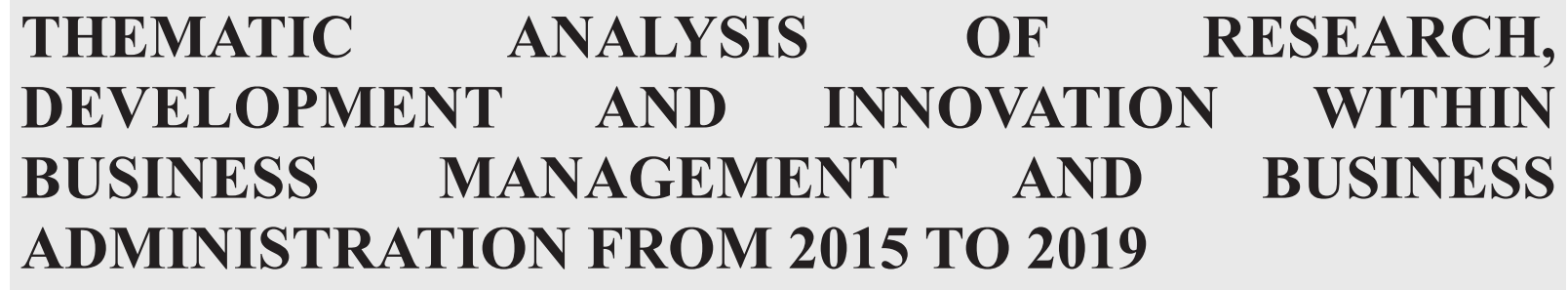

Ana Luisa Hernández-Gutierrez (Universidad Autónoma de Zacatecas) ${ }^{1 *}$

José Rogelio Hernández-Ponce ${ }^{1 * *}$

José Ricardo López-Robles ${ }^{1 * *}$

\section{Resumen}

La Gestión Empresarial, al igual que otros ámbitos organizacionales, se encuentra en constante evolución, motivado por los cambios científico-tecnológicos y dinámicas empresariales. En la actualidad, encontramos que las organizaciones se enfrentan a escenarios cada vez más complejos y cambiantes que requieren del uso intensivo de datos, información y conocimientos en todos sus procesos, especialmente aquellos que tienen que ver con las actividades estratégicas como son los procesos de Investigación, Desarrollo e Innovación. Esto conlleva para las organizaciones el diseño, desarrollo, despliegue y mantenimiento de modelos de gestión avanzados, que aseguren la competitividad tanto a nivel local, como regional e internacional. En este sentido, el presente trabajo busca analizar la gestión de la Investigación, Desarrollo e Innovación en el área de la Gestión Empresarial dentro de las Organizaciones, a través del uso de técnicas y herramientas bibliométricas, como son los mapas científicos usando SciMAT. Para ello, todos los documentos disponibles entre 2015 y 2019 que guarden relación con estas áreas se recabaron de la base de datos Scopus, a fin de identificar las principales temáticas y su evolución, así como los autores, organizaciones, países y fuentes más productivas. Finalmente, este análisis establece un marco de referencia que permite a las organizaciones aumentar su competitividad a través de una metodología de mejora continua de la Investigación, Desarrollo e Innovación de la Gestión Empresarial.

Palabras Clave: Gestión Empresarial; Administración de Empresas; Investigación y Desarrollo; Innovación; Inteligencia Competitiva; Inteligencia Estratégica.

Códigos JEL: M00, M01.

\footnotetext{
${ }^{1}$ Unidad Académica de Contaduría y Administración, Universidad Autónoma de Zacatecas, Contabilidad y Administración S/N, Progreso, 98066 Zacatecas (México).

*Correo-e: analuisa.hernandezgutierrez@unizacatecas.edu.mx

**Correo-e: jrogeliohdez@uaz.edu.mx

***Correo-e: ricardolopezrobles@outlook.com

Autor en correspondencia: José Ricardo López-Robles, ricardolopezrobles@outlook.com

Fecha envío: 31/06/2020. Fecha aceptación: 22/03/2021.
} 


\begin{abstract}
Business Management, like other organizational areas, is constantly evolving, motivated by scientific-technological changes and business dynamics. Currently, we find that organizations face increasingly complex and changing scenarios that require the intensive use of data, information, and knowledge in all their processes, especially those that have to do with strategic activities such as Research processes, Development and Innovation. For organizations, this entails the design, development, deployment, and maintenance of advanced management models, ensuring competitiveness locally, regionally, and internationally. Hence, the present study analyzes the management of Research, Development, and Innovation from the perspective of Business Management within Organizations, through the use of bibliometric techniques and tools, such as SciMAT scientific maps. For this, all the available documents between 2015 and 2019 that are related to these areas, were collected from the Scopus database, in order to identify the main topics and their evolution, as the most productive authors, organizations, countries, and sources. Finally, this analysis establishes a reference framework allowing organizations to increase their competitiveness through a methodology of continuous improvement of Research, Development, and Innovation in Business Management.
\end{abstract}

Keywords: Business Management; Business Administration; Research and Development; Innovation; Competitive Intelligence; Strategic Intelligence.

JEL Codes: M00, M01.

\title{
1. INTRODUCCIÓN
}

Actualmente, las organizaciones se ven envueltas en desafíos económicos, tecnológicos, políticos y sociales, los cuales han dejado en entrevisto que para que las organizaciones sean competitivas, deben pasar de una posición reactiva a una posición proactiva, con capacidad para innovar y adaptarse ágilmente a las singularidades que puedan suscitarse, utilizando nuevas estrategias, en todas sus áreas (López-Robles et al., 2020).

Los estudios relacionados con el ámbito de la Investigación, Desarrollo e Innovación $(\mathrm{I}+\mathrm{D}+\mathrm{i})$ han ido migrando de estar centrados exclusivamente en áreas técnicas y productivas, a considerar las relaciones de las organizaciones con el entorno, donde proveedores, clientes, competidores, agentes científicos, académicos y públicos, entre otros, son fundamentales en la generación y transferencia de conocimiento, así como en el aprendizaje organizacional y, por consiguiente, de las capacidades organizacionales para la innovación, entendida como proceso y resultado (Becerra-Rodriguez et al., 2013).

Los conceptos de Gestión y Administración Empresarial, han evolucionado en la misma medida que las organizaciones, hacia nuevas tecnologías y en nuevos mercados con el fin de satisfacer las cada vez mayores exigencias de los usuarios en términos de productos y servicios. Asimismo, la Gestión y Administración Empresarial ya no son argumentos de carácter regional, en la evolución de nueva sociedad de información y conocimiento, a estos cambios debe considerarse ahora como un asunto de índole global, para desarrollo de nuevas estrategias para la permanencia en el mercado y satisfacciones a cliente en cualquier parte del mundo (Hernandez-Palma, 2011).

Para entender en mejor medida la situación anterior, se aplicó la bibliometría en éste estudio, a través el uso de mapas científicos y análisis de desempeño, las cuales son herramientas comúnmente utilizadas para analizar la literatura, siendo éstas cada vez más recurridas para medir la productividad y la calidad académica y científica (López-Robles, 2019a). 
Esta investigación tiene como objetivo sentar las bases que servirán para la integración de los enfoques de Investigación, Desarrollo e Innovación, y Gestión Empresarial y Administración de Empresas dentro de un mismo marco, para así identificar las principales líneas de investigación, autores más productivos y publicaciones más citadas de estos enfoques, así como las tendencias a futuro, y equilibrar sus estructuras intelectuales con el objetivo de poder alinear éstas dentro de las organizaciones. El área de conocimiento de acuerdo a la clasificación de la OCDE, en donde se establece el siguiente artículo, es contundentemente dentro del área social de Economía y Negocios.

El presente artículo se estructura de la siguiente manera: la Sección 2 describe una breve visión sobre el estado del arte y cómo se pretende dar respuesta a la oportunidad de entender las nuevas relaciones que se dan entre Investigación, Desarrollo e Innovación, y Gestión Empresarial. En la Sección 3, se expone la metodología y se especifican los parámetros de búsqueda utilizados para la creación de la base de datos utilizada en esta investigación. En la Sección 4, se exponen los resultados del análisis bibliométrico y las estructuras intelectuales del análisis planteado, dejando por último, en la Sección 5 una discusión sobre las principales conclusiones obtenidas.

\section{LA INVESTIGACIÓN, DESARROLLO E INNOVACIÓN, Y GESTIÓN EMPRESARIAL Y ADMINISTRACIÓN DE EMPRESAS}

Las empresas son entidades productivas que se desenvuelven en ambientes y entornos económicos cada vez más complejos, dentro del mundo de los negocios, y parte de los objetivos de una organización es la consecución de un beneficio a través de la satisfacción de necesidades de mercado, donde se ofertan productos y servicios con la contraprestación de un valor económico, todo ello a través del uso de nuevas estrategias y la utilización de recursos humanos, productivos y organizacionales. En virtud de que las organizaciones son distintas en su composición y desenvolvimiento, el principal recurso de información de estas mismas se basa en la Gestión Empresarial, la cual, les permite existir, prosperar, y generar dinámicas que las lleven a la búsqueda de cómo convertirse en un agente de cambio.

La Administración de Empresas, entendida como el proceso de administrar una organización para que se mantenga estable y continúe creciendo, incluye entre sus características el desempeño o la gestión de las operaciones comerciales y la toma de decisiones, así como la organización eficiente de personas y otros recursos para dirigir las actividades hacia metas y objetivos comunes. Mientras que, por su parte, la Gestión Empresarial se ve como un subconjunto de la administración, específicamente asociado con los aspectos técnicos y operativos de una organización, distinta de las funciones ejecutivas o estratégicas (Sekaran y Bougie, 2016).

En la actualidad, se incorpora el concepto de Investigación, Desarrollo e Innovación $(\mathrm{I}+\mathrm{D}+\mathrm{i})$ a la forma de administración de las organizaciones, y es un concepto que merece especial atención por el desarrollo social, tecnológico, económico y de competitividad que representa para éstas. El interés por la $\mathrm{I}+\mathrm{D}+\mathrm{i}$ tiene dos perspectivas: la entendida como resultado, lo que implica innovaciones de producto o servicio que han sido presentadas, o el uso efectivo en los procesos administrativos dentro de la organización, y la entendida como proceso, donde la atención se centra en las acciones mediante las cuales las nuevas ideas, los objetos y las prácticas son creadas y desarrolladas o reinventadas, o cómo las innovaciones surgen, se desarrollan y terminan (Becerra-Rodriguez et al., 2013).

Una de las lecciones importantes de las últimas dos décadas ha sido el papel fundamental de la $\mathrm{I}+\mathrm{D}+\mathrm{i}$ en el desarrollo económico, donde la creación de capacidades de innovación 
ha jugado un papel central en la dinámica de crecimiento de los países en desarrollo exitosos, y por ende de las organizaciones. La Investigación, Desarrollo e Innovación en las organizaciones puede describirse como un esfuerzo sistemático y organizado para investigar una oportunidad específica, que necesita una solución. Como lo mencionan Sekaran y Bougie (2016), comprende una serie de pasos diseñados y ejecutados con el objetivo de encontrar respuestas a las oportunidades que se presentan respecto a la innovación y competitividad de la organización.

Estos procesos deben llevarse a cabo de manera sistemática, diligente, crítica, objetiva y lógica (Sekaran y Bougie, 2016), ya que los resultados finales esperados serían un descubrimiento que ayude a la gestión y administración de la organización a abordar las oportunidades en las que se ve inmersa.

Otro punto a considerar, son las relaciones verticales y horizontales dentro de redes organizacionales, ya que éstas también promueven el enfoque de $\mathrm{I}+\mathrm{D}+\mathrm{i}$, puesto que las relaciones dentro de las organizaciones, facilitan la introducción de nuevas estrategias y técnicas, mediante la investigación de aspectos derivados de la gestión y administración de empresas (BecerraRodriguez et al., 2013).

Es así que, para integrar estos conceptos resulta de suma importancia conocer sus estructuras intelectuales y la forma en que se relacionan. Por ello, se ha hecho uso de herramientas bibliométricas, las cuales permiten analizar las publicaciones y citas relacionadas en el campo, con el fin de estudiar su impacto y el cómo contribuyen éstas al desarrollo del estado del arte.

La bibliometría estudia los aspectos cuantitativos de la producción académica, científica, su diseminación y uso, por medio de la cual se han desarrollado modelos para realizar pronósticos y tomar decisiones en torno al área de la Gestión empresarial y Administración de Empresas. Los análisis bibliométricos abarcan dos enfoques, primero se centran en analizar la producción de investigación científica a partir de documentos, países, instituciones, revistas y autores. En cuanto al segundo enfoque, el mapeo bibliométrico, permite observar conexiones entre las estructuras de diferentes investigaciones realizadas en el sistema de conocimiento científico, la interrelación de disciplinas y campos de investigación.

La implementación de estos métodos y modelos matemáticos al objeto de estudio (Araújo Ruiz y Arencibia Jorge, 2002), dan soporte a la obtención de un análisis de las regularidades que ofrece el estudio, así como los procesos y las actividades principales del mismo en sus diferentes áreas. Este tipo de análisis se considera objetivo y confiable, lo que permite a los investigadores obtener información adicional no presentada por las revisiones de la literatura tradicional. Por tanto, no es un sustituto sino un complemento de la investigación académica.

\section{METODOLOGÍA}

Para analizar de qué forma interactúan la Investigación, Desarrollo e Innovación, y Gestión Empresarial y Administración de Empresas se propone establecer un contexto común para el desarrollo de estos a través del análisis bibliométrico de estos conceptos y la visualización de un mapa científico conjunto.

Para ello, se decidió evaluar el rendimiento y relación de estas áreas en conjunto, a través del análisis de los principales indicadores bibliométricos de 2015 a 2019 y la elaboración de mapas científicos utilizando SciMAT (Cobo et al., 2011; Liu et al., 2015), y a la siguiente metodología (ver Figura 1): (1) Recuperación y procesamiento de datos (Cabeza-Ramírez et al., 2018; González-Valiente, 2016; Lagar et al., 2014), (2) Confección y normalización de los mapas científicos (Cantos-Mateos et al., 2013; López-Robles, 2020), y (3) Visualización y 


\section{FIGURA 1. DIAGRAMA DE FLUJO DE LA METODOLOGÍA EMPLEADA.}

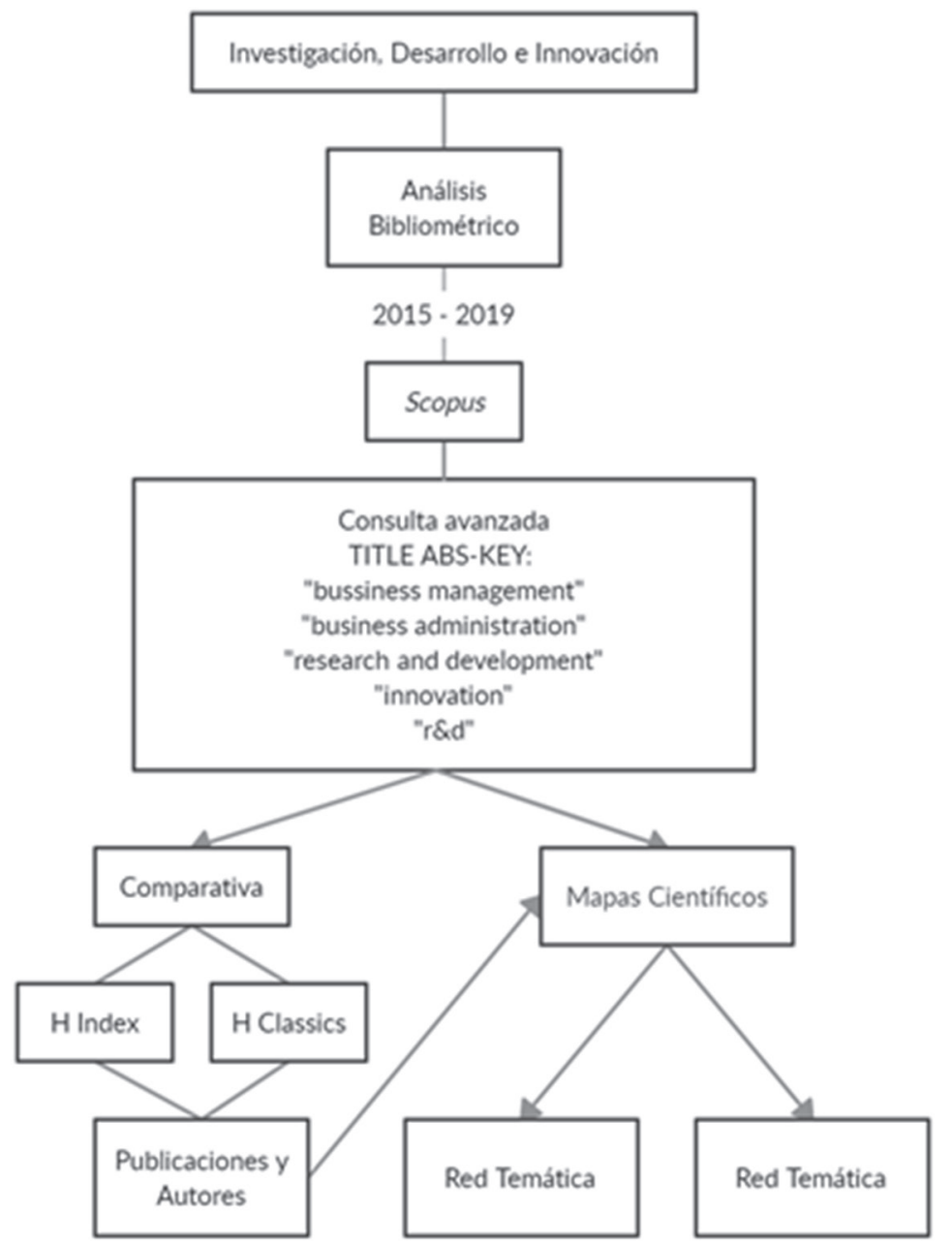

Fuente: Elaboración propia.

análisis de las principales relaciones entre componentes (Cuartas et al., 2014; López-Robles, 2020; Zhao y Strotmann, 2015).

En este caso, los mapas tienen por objetivo representar de forma espacial cómo las publicaciones a través de las palabras clave se encuentran interrelacionadas. Este método, es utilizado ampliamente también para entender, descubrir y mostrar relaciones ocultas o difíciles de ver entre temas de interés para el desarrollo de un área o campo de conocimiento.

Además, a través del mapa estratégico, se caracteriza el periodo analizado a manera de red temática y se representa como un conjunto de temas clasificados y posicionados en un plano dividido en cuatro categorías según los indicadores de centralidad y densidad de Callon (Callon et al., 1991). La centralidad mide el grado de interacción de una red con respecto a otras redes en base a las palabras clave, reflejando con ello el grado de fuerza de los enlaces externos entre temas. Esta medida se puede interpretar como la importancia de un tema en el desarrollo del campo de conocimiento analizado o el grado de cohesión que existe con otros temas. Por su parte, la densidad, mide la fuerza interna de una red atendiendo a los enlaces que describen un tema. 
Atendiendo al primer punto de la metodología planteada se recuperaron los documentos disponibles sobre Investigación, Desarrollo e Innovación, y Gestión Empresarial y Administración de Empresas desde 2015 hasta 2019 de la base de datos Scopus. Estos documentos y su información bibliográfica, como son el título, autores, afiliación, año de publicación, país, revista, citas recibidas, entre otros, han sido recopilados en texto plano el 7 de mayo de 2020, mediante la siguiente consulta avanzada: TITLE-ABS-KEY ("business management" OR "business administration") AND TITLE-ABS-KEY ("research and development" OR "innovation" OR "r\&d").

Las consultas y mapas científicos han sido realizados valiéndose de conceptos en inglés, atendiendo al hecho de que los documentos se encuentran mayormente publicados en dicho idioma. Los documentos presentan título, resumen y palabras clave en inglés, por lo que podemos insistir en que este análisis cubre la totalidad de la literatura existente. Cabe resaltar que las búsquedas han sido limitadas hasta el año 2019, atendiendo al hecho de contar con datos comparables con años completos.

Se han revisado todos los registros recuperados para garantizar su pertinencia y homogeneidad, examinando los documentos completos para su clasificación, y evitar que un documento, autor o cualquier otro elemento pudiera aparecer con dos o más variantes, especialmente en los casos en los que se registró alguna diferencia o falta de información.

En términos de rendimiento bibliométrico, los indicadores utilizados resaltan la productividad de los autores en términos de publicaciones y citas, países, organizaciones y fuentes de información, principalmente. Además, se generó una comparativa común entre las publicaciones y los autores, y se evaluaron los resultados aplicando el h-index y H-Classics (Alonso et al., 2009; Hirsch, 2005; López-Robles, 2020; Martínez et al., 2014; Schreiber, 2007; Schubert, 2007).

Finalmente, para la observación de los mapas científicos se recurrió a la herramienta bibliométrica SciMAT, lo que permitió la definición de una estructura conceptual conjunta y el análisis de sus relaciones directas e indirectas (Callon et al., 1991; González-Valiente, 2016; He, 1999; López-Robles, 2020; Sternitzke y Bergmann, 2009).

\section{ANÁLISIS DEL RENDIMIENTO OBTENIDO}

En esta sección, se analiza el rendimiento de la Investigación, Desarrollo e Innovación, y Gestión Empresarial y Administración de Empresas, en términos de publicaciones y áreas de estudio, fuentes de información, citas, distribución geográfica, y autores más productivos. También, los mapas científicos donde se constituyen las áreas temáticas más representativas, su composición, relación y evolución, utilizando SciMAT.

\subsection{Publicaciones, citas, tipología de publicaciones, fuentes de información, áreas de investigación y distribución geográfica}

Tomando en cuenta la consulta realizada, se recuperaron un total de 255 documentos en el periodo de 2015 a 2019. En la Figura 2 se muestra la distribución anual de las citas y las publicaciones relacionadas con las áreas de Investigación y Desarrollo e Innovación, Gestión Empresarial y Administración de Empresas dentro del periodo definido.

En términos de producción, se pueden acentuar dos hitos relacionados con el crecimiento de la Investigación y Desarrollo e Innovación, Gestión Empresarial y Administración de Empresas en la literatura. El primer hito, refleja la productividad que se da en el 2016, periodo en el cual se registraron 49 publicaciones, número más alto que incluso años posteriores (en los 
años 2017 y 2018 el número cayó a 47 publicaciones anuales). Mientras que el segundo hito se registra en 2019, cuando se alcanza el valor máximo de publicaciones relacionadas con estos enfoques, con un numero de 67 publicaciones.

\section{FIGURA 2. DISTRIBUCIÓN ANUAL DE PUBLICACIONES Y CITAS ALOJADAS EN SCOPUS DE 2015-2019.}

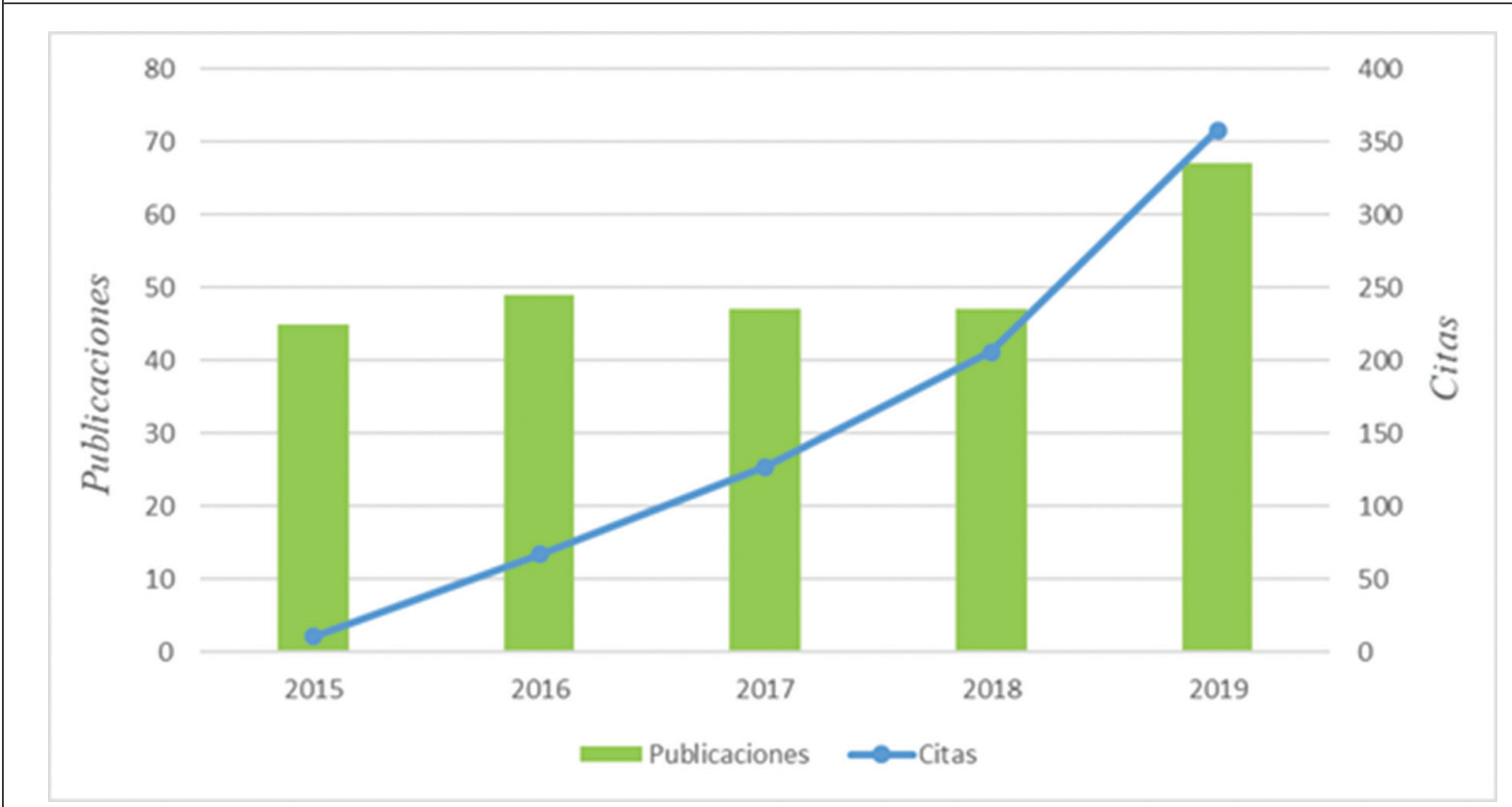

Fuente: Elaboración propia.

En términos de tipología de documentos, la consulta se compone principalmente de Artículos (157), Publicaciones de congresos (87), y Revisiones del Estado del Arte (11). El de mayor relevancia corresponde a los artículos reflejo de la participación de grupos académicos, y a pesar de que los congresos también son foros adecuados para los grupos, suelen tener un enfoque ligeramente más abierto, situación que se refleja en su desarrollo e interés.

En esta misma figura, se muestra la distribución de las citas recibidas por las publicaciones asociadas la Investigación y Desarrollo e Innovación, Gestión Empresarial y Administración de Empresas según la base de datos Scopus al 7 de mayo de 2020.

Al igual que el caso de las publicaciones, la distribución de las citas muestra también una tendencia positiva de crecimiento de 2015 a 2019. Durante este período, se registraron 769 citas para estas áreas. Después de haber tenido una tendencia creciente hasta 2019, donde se alcanzó un máximo de 358 citas, se observa que, las citas han mantenido un crecimiento constante.

Por otro lado, en las áreas de investigación cubiertas por la Investigación y Desarrollo e Innovación, Gestión Empresarial y Administración de Empresas desde 2015 a 2019 no existe apenas variación, siendo los más notables en conjunto: Negocios, Gestión y Contabilidad (141), Ciencias sociales (63), Ingeniería (56), Informática (50), Ciencias de la decisión (40), Economía, Econometría y Finanzas (39), Energía (17), Ciencias ambientales (17) y Matemáticas (11). Cabe mencionar que las cuatro principales fuentes de información son: Sustainability Switzerland (8), Journal of Business Research (6), Advances in Intelligent Systems and Computing (4), Management Research Review (4). 
Por último, los países más productivos tomando en cuenta la búsqueda son Estados Unidos (24), China (23), España (22), Rusia (18), Reino Unido (17), India (14), Brasil (11), Rumania (11), Taiwán (10) y Corea del Sur (9). Estos países tienen una apuesta por los ámbitos de la $\mathrm{I}+\mathrm{D}+\mathrm{i}$, lo cual desarrolla su especialización en el área. Cabe resaltar que hay un total de 64 países que se relacionan con las publicaciones asociadas a la Investigación y Desarrollo e Innovación, Gestión Empresarial y Administración de Empresas.

\subsection{Autores más productivos y autores más citados}

Como se ha visualizado, la Investigación y Desarrollo e Innovación, Gestión Empresarial y Administración de Empresas presenta una actividad constante y en crecimiento, características fundamentales al momento de estipular la evolución y consolidación de las áreas de conocimiento que cubren, y es por ello que, para complementar lo anterior, a continuación, se identifican los autores más productivos y citados.

En la Tabla 1 se muestran los autores más productivos en los campos de la Investigación, Desarrollo e Innovación, y la Gestión Empresarial y Administración de empresas en el periodo de 2015 a 2019. Dentro de los autores más productivos destaca Gumerova, G.I. que se encuentra como el autor más productivo por la cantidad de publicaciones presentadas ( 3 publicaciones).

TABLA 1. AUTORES MÁS PRODUCTIVOS EN EL CAMPO DE I+D+I Y LA GESTIÓN EMPRESARIAL Y ADMINISTRACIÓN DE EMPRESAS DE 2015 A 2019

(Publicaciones, $\mathrm{n}=255$ ) Autor(es)

(3) Gumerova, G. I.

(2) Batkovskiy, A. M.; Fomina, A. V.; Gavril, R. M.; Grab, B.; Kalachikhin, P. A.; Lis, A.; Mateiu, A.; Olaru, M.; Safiullin, M. R.; Santoso, H.; Scheepers, C.; Semenova, E. G.; Shaimieva, E. S.; Sudolska, A.; Telnov, Y. F.; Tseng, M. L.

Fuente: Elaboración propia.

En algunos puestos de la tabla encontramos empates en la cantidad de publicaciones de distintos autores, por lo que se ha procedido a incluirlos en orden alfabético. Cabe mencionar que, además de los presentados en la tabla, se cuenta con 143 autores más que cuentan con una única publicación según la búsqueda realizada.

Por último, en la Tabla 2 se muestran los autores más citados en el campo de la Investigación y Desarrollo e Innovación, Gestión Empresarial y Administración de Empresas entre 2015 y 2019. Es importante resaltar, que, dentro de los autores más citados, Tseng M.-L es el único autor que aparece dentro de los autores más productivos y más citados a la vez. 


\section{TABLA 2. AUTORES MÁS CITADOS EN EL CAMPO DE I+D+I Y LA GESTIÓN} EMPRESARIAL Y ADMINISTRACIÓN DE EMPRESAS DE 2015 A 2019

\begin{tabular}{|l|}
\hline (Citas, n=769) Autor(es) \\
\hline (69) Abazi-Alili H.; Dana L. P.; Gerguri-Rashiti S.; Ramadani V.; Ratten V. \\
\hline (43) Tseng M. L. \\
\hline (40) Prause G. \\
\hline (32) Barmuta K. A.; Dubrova L. I.; Kobersy I. S.; Muradova S. S.; Shkurkin D. \\
\hline (28) Coles T.; Dinan C.; Warren N. \\
\hline (27) Dharsuky A.; Jumilawati E.; Muda I.; Sihombing M. \\
\hline (24) Bui T. D. \\
\hline (23) Boronat-Moll C.; Hervas-Oliver J. L.; Rojas R.; Sempere-Ripoll F. \\
\hline (22) Charterina J.; Kaur G.; Khanam Quareshi T.; Pando-Garcia J.; Perianez-Canadillas I. \\
\hline (21) Pizam A.; Rivera M. A. \\
\hline (19) Chou P. J.; Liao C. J.; Wu K. J. \\
\hline (18) Jun S. P.; Park D. H. \\
\hline (17) Brones F. A.; Carvalho M. M. D.; Zancul E. D. S. \\
\hline
\end{tabular}

Fuente: Elaboración propia.

\subsection{Análisis de publicaciones más citadas}

Tomando en cuenta los resultados previos, a continuación se consideran las publicaciones más relevantes y más citadas para el enfoque de Investigación y Desarrollo e Innovación, Gestión Empresarial y Administración de Empresas por medio del uso del h-index y H-Classics (Hirsch, 2005; López-Robles, 2020; Martínez et al., 2014).

Se analiza la importancia, presencia y peso de las publicaciones más citadas en el desarrollo de la Investigación y Desarrollo e Innovación, Gestión Empresarial y Administración de Empresas desde la composición de dos indicadores: h-index y H-Classics (Martinez et al., 2015; Ale Ebrahim et al., 2014).

Por un lado, el h-index nos permite observar la productividad y el impacto de un grupo de publicaciones, a través de la relación que existe el número de artículos y las citas de estos (Hirsch, 2005). Por otro lado, el H-Classics sirve de indicador de control, ya que permite estandarizar el procedimiento de búsqueda de las citaciones de los artículos incluidos en el h-index (LópezRobles, 2020; Martínez et al., 2014). La combinación de los dos índices reduce la cantidad de diferencias que puede existir entre los artículos más citados y lo que no han logrado obtener la misma profundidad de conocimiento científico, estableciendo un punto de referencia común para las publicaciones referentes dentro de un área de investigación.

Es por ello, que se utilizó como referencia el $h$-index indicado por Scopus para la consulta, para así, identificar las publicaciones más relevantes en el desarrollo de la Investigación y Desarrollo e Innovación, Gestión Empresarial y Administración de Empresas. Según el h-index de la consulta, ésta se compone por 15 publicaciones entre artículos, publicaciones en congresos, revisiones y libros, y su distribución y la cantidad de citas acumuladas, se muestra en la Figura 3. Finalmente, el listado H-Classics se presenta en la Tabla 3. 


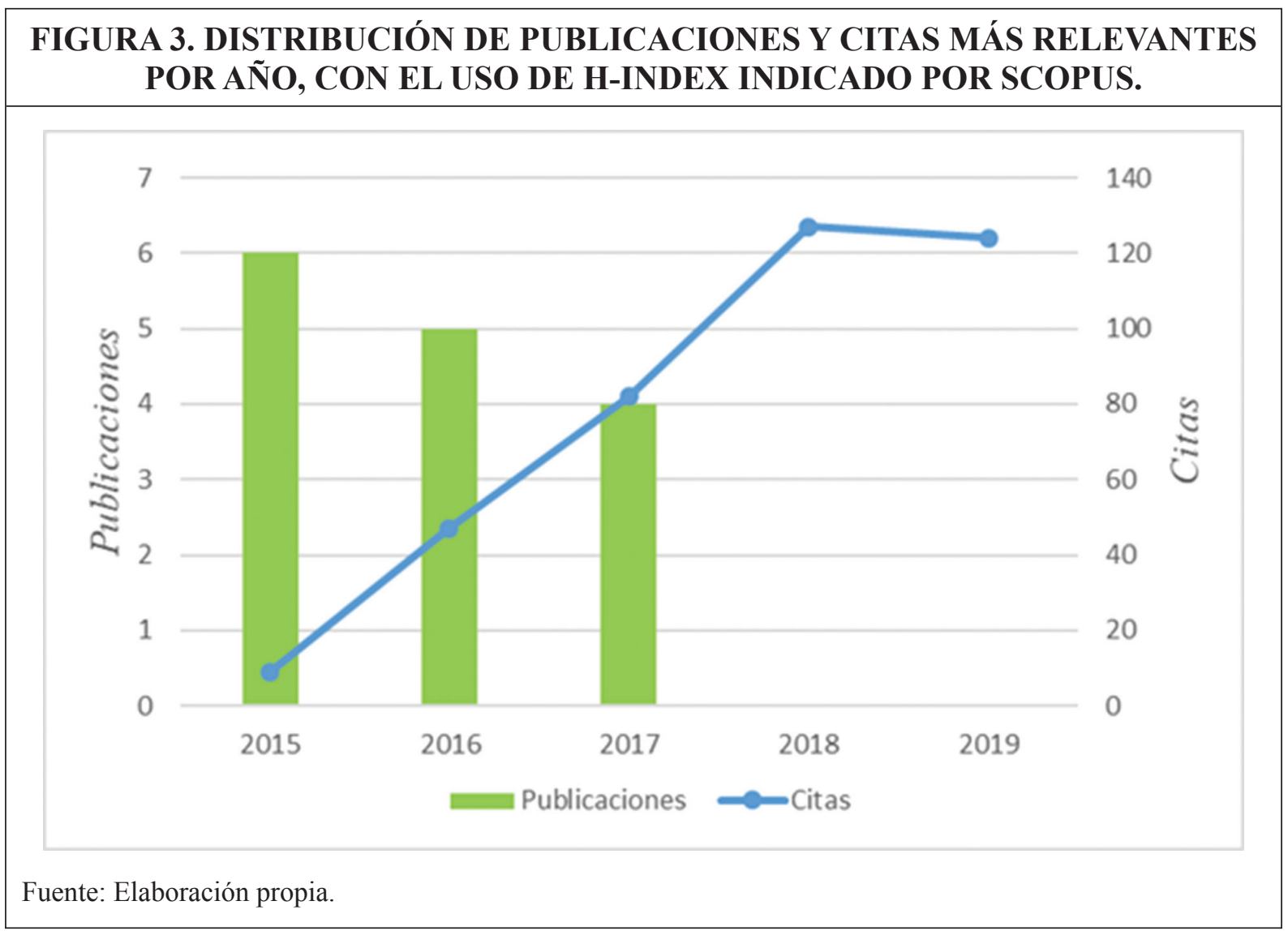

\section{TABLA 3. H-CLASSICS PARA LA INVESTIGACIÓN, DESARROLLO E INNOVACIÓN Y LA GESTIÓN EMPRESARIAL Y ADMINISTRACIÓN DE EMPRESAS DE 2015 A 2019}

\begin{tabular}{|c|l|l|}
\hline No. & Titulo (Autor(es), año) & Citas \\
\hline 1 & $\begin{array}{l}\text { ICT, Innovation and Firm Performance: The Transition Economies } \\
\text { Context (Gerguri-Rashiti S., Ramadani V., Abazi-Alili H., Dana L. P., } \\
\text { Ratten V., 2017) }\end{array}$ & 79 \\
\hline 2 & $\begin{array}{l}\text { Sustainable business models and structures for industry 4.0 (Prause G., } \\
2015)\end{array}$ & 47 \\
\hline 3 & $\begin{array}{l}\text { Identifying eco-innovation in industrial symbiosis under linguistic } \\
\text { preferences: A novel hierarchical approach (Tseng M. L., Bui T. D., } \\
\text { 2017) }\end{array}$ & 35 \\
\hline 4 & $\begin{array}{l}\text { The system of the methodological principles of management of } \\
\text { enterprise development (Kobersy I. S., Barmuta K. A., Muradova S. S., } \\
\text { Dubrova L. I., Shkurkin D., 2015) }\end{array}$ & 32 \\
\hline 5 & $\begin{array}{l}\text { Energy practices among small- and medium-sized tourism enterprises: } \\
\text { A case of misdirected effort? (Coles T., Dinan C., Warren N., 2016) }\end{array}$ & 30 \\
\hline 6 & $\begin{array}{l}\text { Factors affecting the success of local innovation systems with } \\
\text { government programs as moderators (Sihombing M., Muda I., } \\
\text { Jumilawati E., Dharsuky A., 2017) }\end{array}$ & 28 \\
\hline
\end{tabular}




\begin{tabular}{|c|l|l|}
\hline 7 & $\begin{array}{l}\text { Business simulation games with and without supervision: An analysis } \\
\text { based on the TAM model (Pando-Garcia J., Perianez-Canadillas I., } \\
\text { Charterina J., 2016) }\end{array}$ & 26 \\
\hline 8 & $\begin{array}{l}\text { Factors obstructing intentions to trust and purchase products online } \\
\text { (Kaur G., Khanam Quareshi T., 2015) }\end{array}$ & 25 \\
\hline 9 & $\begin{array}{l}\text { Technological innovation without R\&amp;D: unfolding the extra gains } \\
\text { of management innovations on technological performance (Hervas- } \\
\text { Oliver J. L., Sempere-Ripoll F., Boronat-Moll C., Rojas R., 2015) }\end{array}$ & 25 \\
\hline 10 & $\begin{array}{l}\text { Advances in hospitality research: “from Rodney Dangerfield to Aretha } \\
\text { Franklin" (Rivera M. A., Pizam A., 2015) }\end{array}$ & 24 \\
\hline 11 & $\begin{array}{l}\text { Understanding innovation for sustainable business management } \\
\text { capabilities and competencies under uncertainty (Wu K. J., Liao C. J., } \\
\text { Tseng M. L., Chou P. J., 2015) }\end{array}$ & 22 \\
\hline 12 & $\begin{array}{l}\text { Reviews, action and learning on change management for eco-design } \\
\text { transition (Brones F. A., Carvalho M. M. D., Zancul E. D. S., 2017) }\end{array}$ & 21 \\
\hline 13 & $\begin{array}{l}\text { Consumer information search behavior and purchasing decisions: } \\
\text { Empirical evidence from Korea (Jun S. P., Park D. H., 2016) }\end{array}$ & 18 \\
\hline 14 & $\begin{array}{l}\text { Sense-making, Sense-Censoring and Strategic Inaction: The Discursive } \\
\text { Enactment of Power and Politics in a Multinational Corporation } \\
\text { (Whittle A., Mueller F., Gilchrist A., Lenney P., 2016) }\end{array}$ & 15 \\
\hline 15 & $\begin{array}{l}\text { Conceptualizing public innovative capacity: A framework for } \\
\text { assessment (Gieske H., van Buuren A., Bekkers V., 2016) }\end{array}$ & 15 \\
\hline
\end{tabular}

La distribución de publicaciones muestra que el año 2015 tuvo las publicaciones más relevantes con un numero de 6 publicaciones consideradas en el $h$-index, representa el 40 por ciento de la consulta; para los años 2016 y 2017 se despliegan 5 y 4 publicaciones, que representan el 33.3 por ciento y 26 por ciento del total de la consulta, respectivamente. En términos de citas, las publicaciones identificadas acumulan 389 referencias, lo que representa el 50.5 por ciento de las citas totales acumuladas en la consulta. Para el año 2019, las citas disminuyen de 127 a 124 citas, las cuales representan un 22 por ciento de las publicaciones de dicha consulta (López-Robles et al., 2020).

Se puede observar que estos aspectos coinciden con la evolución de producción y citas observados con anterioridad, y se mantienen una vez transcurrido un periodo de cuatro años, lo que sugiere que el progreso y fortalecimiento de estas publicaciones como parte del núcleo del de la Investigación y Desarrollo e Innovación, Gestión Empresarial y Administración de Empresas (Wang, 2013; López-Robles et al., 2020).

De igual forma, la productividad de los autores muestra un comportamiento uniforme, ya que de los 15 autores que respaldan las publicaciones más relevantes, tan solo uno de ellos cuenta con dos publicaciones, siendo el autor más productivo Tseng M.-L.; mientras que el resto de los autores registra una única publicación.

En términos de distribución geográfica, los países de España, Taiwán, Reino Unido y Países Bajos figuran para la posición principal con 2 publicaciones cada uno, seguidos por Australia, Brasil, Colombia, Estonia, India, Indonesia, Kuwait, Macedonia del Norte, Rusia, Corea de Sur y Estados Unidos, con 1 publicación respectivamente. Esto contrasta con los resultados iniciales donde países como Estados Unidos, China y Rusia aparecían en las primeras posiciones. 
Continuando en esta línea, observamos que la organización más productiva dentro de las publicaciones más relevantes es LungHwa University of Science and Technology con 2 publicaciones.

Por otro lado, las principales áreas de investigación abarcadas por la Investigación y Desarrollo e Innovación, Gestión Empresarial y Administración de Empresas desde 2015 a 2019 en conjunto, coinciden con las identificadas en puntos anteriores, aunque su prioridad varia ligeramente posicionado Negocios, Administración y Contabilidad, delante de Energía, Ciencias Sociales, Ciencias Ambientales, Ingeniería, Economía, Econometría y Finanzas, Artes y Humanidades, Ciencias de la Decisión, y Psicología.

Otra situación distinta ocurre con las principales fuentes de información, en las cuales la revista Journal of Cleaner Production se sitúa como la primera fuente con 3 publicaciones, seguida con 1 publicación por: Asia Pacific Journal of Marketing and Logistics, Innovation Journal, International Journal of Contemporary Hospitality Management, International Journal of Economic Research, Journal of Business Research, Journal of Security and Sustainability Issues, Mediterranean Journal of Social Sciences, Organization Studies, Sustainability Switzerland, Technological Forecasting and Social Change, Technology Analysis and Strategic Management, y Thunderbird International Business Review.

\subsection{Análisis del mapa de evolución de los enfoques de inteligencia}

Continuando con la metodología descrita, la Figura 4 proporciona una visión general del mapeo científico, su desempeño y las relaciones entre los temas principales en Investigación y Desarrollo e Innovación, Gestión Empresarial y Administración de Empresas de 2015 a 2019.

De acuerdo con el diagrama estratégico, durante este período analizado, la investigación se centró en nueve temas: BUSINESS-MANAGEMENT (gestión empresarial), PRODUCT-DESIGN-AND-DEVELOPMENT (diseño y desarrollo de producto), INTELLECTUAL-PROPERTY-AND-INTELLECTUAL-CAPITAL (propiedad y capital intelectual), STRATEGIC-MANAGEMENT (gestión estratégica), INDUSTRIALMANAGEMENT (gestión industrial), ORGANIZATIONAL-COMPETITIVENESS (competitividad organizacional), BUSSINESS-PERFORMANCE (rendimiento empresarial), ENTREPRENEURSHIP (emprendimiento) y KNOWLEDGE-MANAGEMENT (gestión del conocimiento).

Las medidas de rendimiento de los temas principales presentados en la Figura 4 muestran el número de documentos, citas, citas promedio y h-index por tema. Los más productivos se incluyen en los Temas motor BUSINESS-MANAGEMENT (55); Temas básicos y transversales de ORGANIZATIONAL-COMPETITIVENESS (44) y KNOWLEDGE-MANAGEMENT (26); y Temas emergentes ENTREPRENEURSHIP (32). Sin embargo, los temas más citados (más de 100 citas) están incluidos en Temas motor BUSINESS-MANAGEMENT (139) y Temas básicos y transversales ORGANIZATIONAL-COMPETITIVENESS (128) y KNOWLEDGEMANAGEMENT (102).

En los conceptos más productivos y más citados, los temas son los mismos, (con excepción de ENTREPRENEURSHIP), lo que significa que los temas principales son la referencia de los temas más productivos y más citados. 


\section{FIGURA 4. DIAGRAMA ESTRATÉGICO DE RENDIMIENTO DE LOS TEMAS EN EL PERIODO DE 2015 A 2019 ELABORACIÓN PROPIA CON INFORMACIÓN OBTENIDA DE LA METODOLOGÍA DESCRITA.}

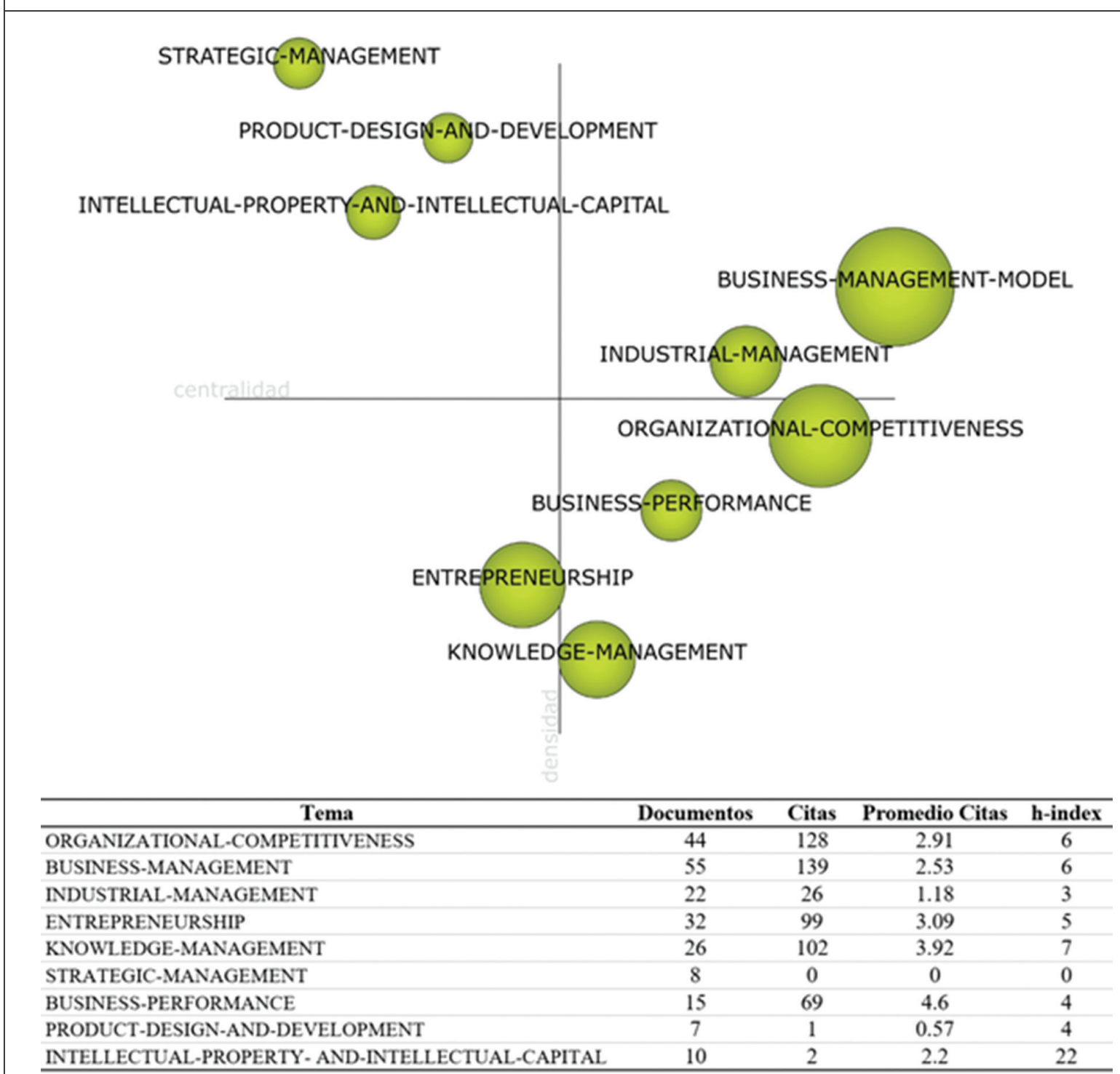

Fuente: Elaboración propia.

Por otro lado, las redes temáticas de cada tema se muestran en la Figura 5. En términos de productividad, el tema más productivo de la Investigación y Desarrollo e Innovación, Gestión Empresarial y Administración de Empresas es el de BUSINESS-MANAGEMENT (gestión empresarial) (Figura 5. (f)), que se relaciona principalmente con HUMAN-RESOURCESMANAGEMENT (gestión de recursos humanos), TEACHING-AND LEARNING-PROCESS (proceso de enseñanza y aprendizaje), BUSINESS-MODEL (modelo de negocios), y VIRTUALLEARNING-ENVIRONMENT (ambiente de aprendizaje virtual). Teniendo en cuenta que este tema está asignado en el Cuadrante 1, su papel es motor para el resto de los temas.

El tema de ORGANIZATIONAL-COMPETITIVENESS (competitividad organizacional) (Figura 5. (g)) es el segundo clasificado en términos de productividad y citas obtenidas. Se relaciona principalmente con temas tales como COMPETITIVE-ADVANTAGE (ventaja 


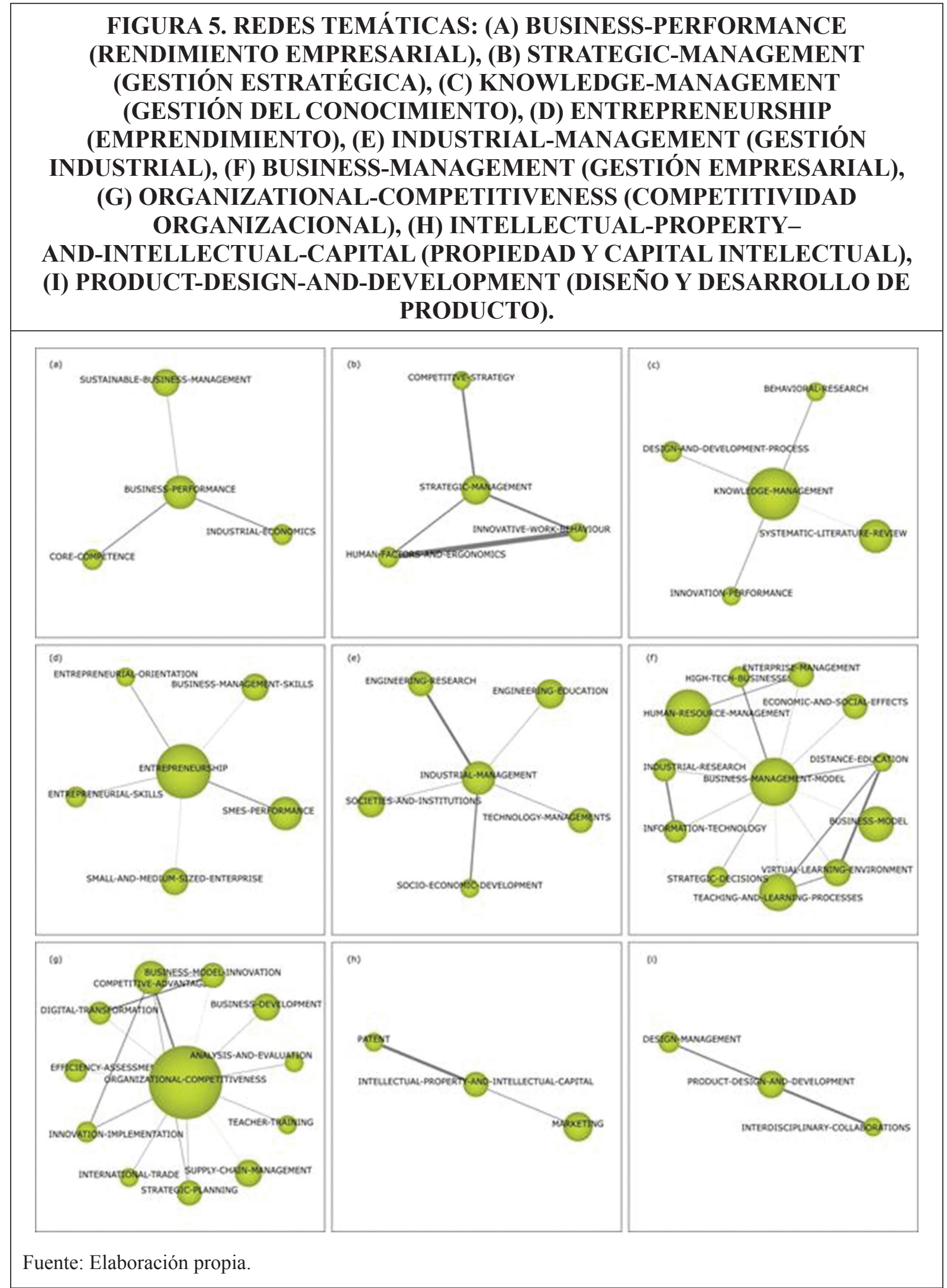


competitiva), BUSINESS-MODEL-INNOVATION (innovación de modelo de negocios), DIGITAL-TRANSFORMATION (transformación digital), BUSINESS-DEVELOPMENT (desarrollo de negocios), y SUPPLY-CHAIN-MANAGEMENT (gestión de cadena de suministros). Este tema se muestra presente en el Cuadrante 4, por lo que se considera Tema básico y transversal para con el resto de los temas.

En un caso similar, ENTREPRENEURSHIP (emprendimiento) se asigna en el cuadrante 3 considerándose Temas emergentes; y KNOWLEDGE-MANAGEMENT (gestión del conocimiento) se asigna en el cuadrante 4, donde fungen como Temas básico y transversal, en base al cuadrante donde se encuentran. El tema ENTREPRENEURSHIP (Figura 5. (d)) se relaciona principalmente con temas como BUSINESS-MANAGEMENTSKILLS (habilidades de gestión de negocios), SMES-PERFORMANCE (rendimiento de PyMEs) y SMALL-AND-MEDIUM-SIZED-ENTRERPRISE (pequeñas y medianas empresas); así mismo, el tema KNOWLEDGE-MANAGEMENT (Figura 5. (c)) está vinculado principalmente a SYSTEMATIC-LITERATURE-REVIEW (revisión sistemática de literatura), INNOVATION-PERFORMANCE (rendimiento de la innovación), DESIGN-ANDDEVELOPMENT-PROCESS (proceso de diseño y desarrollo) y BEHAVIORAL-RESEARCH (investigación del comportamiento).

En la actualidad, la Gestión Empresarial y la Administración De Empresas son temas fundamentales a nivel global, estos conceptos los encontramos en contextos políticos, tecnológicos, sociales, culturales y empresariales y son quienes nos ayudan a identificar y determinar las estrategias ideales para las organizaciones que buscan crecimiento, permitiéndoles afrontar nuevos desafíos sobre la competitividad, los cuales permitirán sobrevivir al cambio constante y a las nuevas exigencias sociales.

Al sentar los conceptos claves como base para el estudio de esta disciplina, podremos identificar las oportunidades de estudio de dichas áreas, como en BUSINESS-PERFORMANCE (Figura 5. (a)), INDUSTRIAL-MANAGEMENT (Figura 5 (e)), y KNOWLEDGEMANAGEMENT (Figura 5. (c)); las cuales son áreas presentes en las bases de la investigación científica, pero por el número de productos y citas, podemos observar que aún se puede ahondar como una línea futura de investigación.

Finalmente, de acuerdo con los resultados obtenidos y teniendo en cuenta los principales temas de investigación relacionados con los temas más citados, es posible afirmar que las publicaciones y los temas de investigación cubiertos por la Investigación y Desarrollo e Innovación en Gestión Empresarial y Administración de Empresas, son sólidos, se encuentran en total vinculación y cuentan con grandes sinergias entre ellos.

\section{CONCLUSIONES}

En términos de cantidad y calidad, la Investigación y Desarrollo e Innovación, Gestión Empresarial y Administración de Empresas presentan una tendencia positiva de crecimiento y consolidación como áreas de conocimiento, a pesar de registrar variaciones durante los años 2017 y 2018. En base a la cantidad de citas generadas en estos ámbitos de conocimiento, se espera que el interés hacia la investigación y su aplicación por parte de académicos y profesionales en este campo, siga creciente dentro de los próximos años.

Para el análisis de producción y citas, se observaron los autores más productivos y los autores más citados. Estos contextos, alientan el desarrollo del campo de la innovación empresarial, estableciendo por un lado el análisis en base a la producción, desde donde podemos identificar autores, grupos de investigación, organizaciones y la aplicación, desde donde podemos identificar que temas de la Investigación y Desarrollo e Innovación, Gestión 
Empresarial y Administración de Empresas están teniendo un mayor impacto en el desarrollo y consolidación del campo de conocimiento.

Los resultados sobre la distribución geográfica, arrojan un panorama sobre la capacidad productiva empresarial y académica, que tienen actualmente países como Australia, Brasil, Colombia, India, Indonesia, Rusia, Corea de Sur y Estados Unidos, entre otros, por sus altos números de productos científicos en el campo. Así también, se puede resaltar el trabajo que presentan países como España, Taiwán y Reino Unido en el aporte al desarrollo de estas áreas de conocimiento y que les posicionan entre los países más productivos tanto en producción científica como en citas de dichos trabajos.

Asimismo, se puede observar que las publicaciones que conforman actualmente el núcleo del campo de la Investigación y Desarrollo e Innovación, Gestión Empresarial y Administración de Empresas, presentan una tendencia a sentar las bases de la investigación en el campo de la Gestión Empresarial y la Administración de Empresas, puesto que se puede observar una joven investigación en el campo, lo que refuerza la coexistencia de la $\mathrm{I}+\mathrm{D}+\mathrm{i}$ dentro de la Gestión Empresarial y la Administración de Empresas.

Por otro lado, con el análisis del mapa científico se observa como los temas principales comparten temas de investigación, pero a su vez cada uno cuenta con elementos propios que resultan necesarios para el desarrollo del otro, y viceversa. Dentro de las líneas que vinculan el enfoque con los demás temas de investigación, se pueden resaltar tres áreas temáticas: ENTREPRENEURSHIP (emprendimiento), BUSINESS-MANAGEMENT (gestión empresarial), y ORGANIZATIONAL-COMPETITIVENESS (competitividad organizacional) y las aplicaciones de estos temas, principalmente en aspectos relacionados con SMES-PERFORMANCE (rendimiento de PyME), HUMAN-RESOURCE-MANAGEMENT (gestión de recursos humanos), y COMPETITIVE-ADVANTAGE (ventaja competitiva) respectivamente.

Además, visualizando el resultado del análisis en conjunto, podemos proponer que tanto la comunidad académica y científica como la empresarial vinculan la Investigación y Desarrollo e Innovación, Gestión Empresarial y Administración de Empresas, con la competitividad de las organizaciones y al desarrollo e innovación éstas, buscando sentar las bases para la construcción de organizaciones innovadoras, ágiles, flexibles y con capacidades avanzadas para la conceptualización, recopilación, análisis, e implementación de información útil para el desarrollo de las organizaciones, independiente del sector o mercado en el que se desenvuelvan.

Es por ello que este trabajo permite a profesionales e investigadores de la Investigación y Desarrollo e Innovación, Gestión Empresarial y Administración de Empresas, conocer los elementos que se relacionan entre ellos. Así también, ayuda a definir nuevas líneas de investigación futuras, en donde se puede analizar la información en una extensión mayor de rango de estudio, o limitarla hacia los temas principales y su desarrollo con los temas que se conectan.

Finalmente, el estudio demanda un seguimiento de año con año sobre el avance obtenido en el campo de la Investigación y Desarrollo e Innovación, Gestión Empresarial y Administración de Empresas, con el objetivo de apoyar el crecimiento y consolidación de esta área de estudio, lo cual generará un efecto sinérgico entre los conceptos base de esta área de estudio, manteniendo la coexistencia y su crecimiento en el tiempo.

Los autores agradecen a la Universidad Autónoma de Zacatecas, CONACYT-Consejo Nacional de Ciencia y Tecnología (México) y COZCyT Consejo Zacatecano de Ciencia Tecnología e innovación el apoyo para la realización de este estudio. 


\section{BIBLIOGRAFIA}

Ale Ebrahim, Nader, Hadi Salehi, Mohamed Amin Embi, Farid Habibi, Hossein Gholizadeh y Seyed Mohammad Motahar. (2014). 'Visibility and citation impact', International Education Studies, 7: 5. https://doi.org/10.5539/ies.v7n4p120

Alonso, Sergio, Francisco Javier Cabrerizo, Enrique Herrera-Viedma y Francisco Herrera. (2009). 'h-Index: A review focused in its variants, computation and standardization for different scientific fields', Journal of Informetrics, 3: 273-89. https://doi.org/10.1016/j. joi.2009.04.001

Araújo-Ruiz, Juan A. y Ricardo Arencibia Jorge. (2002). 'Informetría, bibliometría y cienciometría: aspectos teórico-prácticos', ACIMED.

Becerra-Rodriguez, Fredy, Hector Mauricio Serna Gomez y Julia Clemencia Naranjo Valencia. (2013). 'Redes empresariales locales, investigación y desarrollo e innovación en la empresa. Cluster de herramientas de Caldas, Colombia', Estudios Gerenciales: 247-57. https://doi.org/10.1016/j.estger.2013.05.013

Cabeza-Ramírez, L Javier, Sandra Ma Sánchez Cañizares y Fernando J Fuentes-García. (2018). 'Characterisation of the classics of entrepreneurship (1968-2016). An analysis based on Web of Science', Revista Española de Documentacion Cientifica, 41: 1-16. https://doi. org/10.3989/redc.2018.2.1488

Callon, M., J. P. Courtial y F. Laville. (1991). 'Co-Word Analysis as a Tool for Describing the Network of Interactions between Basic and Technological Research - The Case of Polymer Chemistry', Scientometrics, 22: 155-205. https://doi.org/10.1007/BF02019280

Cantos-Mateos, Gisela, María de los Ángeles Zulueta-García, Benjamín Vargas-Quesada y Zaida Chinchilla-Rodríguez. (2013). "Estudio comparativo sobre la visualización de redes de co-words a través de los descriptores del Science Citation Index y de Medline." In I Congresso ISKO Espanha e Portugal/XI Congresso ISKO Espanha.

Cobo, M. J., A. G. Lopez-Herrera, E. Herrera-Viedma y F. Herrera. (2011). 'Science Mapping Software Tools: Review, Analysis, and Cooperative Study Among Tools', Journal of the American Society for Information Science and Technology, 62: 1382-402. https://doi. org/10.1002/asi.21525

Cuartas, Gabriel Vélez, Henry Gómez Flórez, Ana Úsuga Ciro y Manuel Vélez Trujillo. (2014). 'Diversidad y reconocimiento de la producción académica en los sistemas de evaluación de la investigación en Colombia', Revista Española de Documentacion Cientifica, 37 : 5. https://doi.org/10.3989/redc.2014.3.1133

González-Valiente, Carlos L. (2016). 'Investigación sobre Bibliotecología, Ciencia de la Información, e Inteligencia Empresarial, a través de las presentaciones a los congresos INFO e IntEmpres: un análisis bibliométrico (2002-2012)', Revista Española de Documentacion Cientifica, 39. https://doi.org/10.3989/redc.2016.4.1377

He, Q. (1999). 'Knowledge discovery through co-word analysis', Library Trends, 48: 133-59.

Hernandez-Palma, Hugo Gaspar. (2011). 'La Gestion Empresarial, un enfoque del siglo XX, desde las teorias administrativas cientifica, funcional, burocratica y de relaciones humanas', Escenarios: 38-51.

Hirsch, Jorge E. (2005). 'An index to quantify an individual's scientific research output', Proceedings of the National academy of Sciences of the United States of America, 102: 16569-72. https://doi.org/10.1073/pnas.0507655102

Lagar Barbosa, María Pilar, María Isabel Escalona Fernández y Antonio Pulgarín Guerrero. (2014). 'Análisis de la interdisciplinariedad en la ingeniería química universitaria española', Revista Española de Documentacion Cientifica, 37: 9. https://doi.org/10.3989/ redc.2014.1.1048 
Liu, Zhigao, Yimei Yin, Weidong Liu y Michael Dunford. (2015). 'Visualizing the intellectual structure and evolution of innovation systems research: a bibliometric analysis', Scientometrics, 103: 135-58. https://doi.org/10.1007/s11192-014-1517-y

López-Robles, J. R., Otegi-Olaso, J. R., Gómez, I. P. y Cobo, M. J. (2019). ‘30 years of intelligence models in management and business: A bibliometric review', International journal of information management, 48: 22-38. https://doi.org/10.1016/j.ijinfomgt.2019.01.013

López-Robles, J. R., Otegi-Olaso, J. R., Porto-Gomez, I., Gamboa-Rosales, H. y GamboaRosales, N. K. (2020). 'Understanding the intellectual structure and evolution of Competitive Intelligence: A bibliometric analysis from 1984 to 2017', Technology Analysis \& Strategic Management, 5: 604-19. https://doi.org/10.1080/09537325.2019 .1686136

Martinez, M. A., M. J. Cobo, M. Herrera y E. Herrera-Viedma. (2015). 'Analyzing the Scientific Evolution of Social Work Using Science Mapping', Research on Social Work Practice, 25: 257-77. https://doi.org/10.1177/1049731514522101

Martinez, Miguel Angel, Manuel Herrera, Javier López-Gijón y Enrique Herrera-Viedma. (2014). 'H-Classics: Characterizing the concept of citation classics through H-index', Scientometrics, 98: 1971-83. https://doi.org/10.1007/s11192-013-1155-9

Schreiber, Martin. (2007). 'Self-citation corrections for the Hirsch index', EPL (Europhysics Letters), 78: 30002. https://doi.org/10.1209/0295-5075/78/30002

Schubert, András. (2007). 'Successive h-indices', Scientometrics, 70: 201-05. https://doi. org/10.1007/s11192-007-0112-x

Sekaran, Uma, and Roger Bougie. 2016. Research Methods For Business: A Skill Building Approach (Wiley: Chichester, West Sussex, UK.).

Sternitzke, C. y I. Bergmann. (2009). 'Similarity measures for document mapping: Acomparative study on the level of an individual scientist', Scientometrics, 78: 113-30. https://doi. org/10.1007/s11192-007-1961-z

Wang, J. (2013). 'Citation time window choice for research impact evaluation', Scientometrics, 94: 851-72. https://doi.org/10.1007/s11192-012-0775-9

Zhao, Dangzhi y Andreas Strotmann. (2015). 'Analysis and visualization of citation networks', Synthesis lectures on information concepts, retrieval, and services, 7: 1-207. https://doi. org/10.2200/S00624ED1V01Y201501ICR039 